\title{
Identifying the targets of the emotions expressed in health forums
}

\author{
Sandra Bringay ${ }^{1,2}$, Eric Kergosien ${ }^{1,3}$, Pierre Pompidor ${ }^{1}$, and Pascal Poncelet ${ }^{1}$ \\ 1-LIRMM UMR 5506, CNRS, University of Montpellier 2 \\ bringay, kergosien, pompidor, poncelet@lirmm.fr, \\ 2-AMIS, University of Montpellier 3 \\ 3-Maison de la télédétection, IRSTEA
}

\begin{abstract}
In the framework of the French project Patients' Mind, we focus on the semi-automatic analysis of online health forums. Online health forums are areas of exchange where patients, on condition of anonymity, can talk about their personal experiences freely. These resources are a gold mine for health professionals, giving them access to patient to patient exchanges, patient to health professional exchanges and even health professional to health professional exchanges. In this paper, we focus on the emotions expressed by the authors of the messages and more precisely on the targets of these emotions. We suggest an innovative method to identify these targets, based on the notion of semantic roles and using the FrameNet resource. Our method has been successfully validated on real data set.
\end{abstract}

Keywords: Opinion mining, emotion analysis, health system applications.

\section{Introduction}

In the framework of the French project Patients' Mind ${ }^{1}$, we focus on the semiautomatic analysis of online health forums. Online health forums are areas of exchange where patients, on condition of anonymity, can talk about their personal experiences freely. An example is the very active forum healthforum ${ }^{2}$, which allows internet users (often non-health professionals) to exchange their opinions on their health situation. In [1], Hancock and al. demonstrated that the ability to communicate anonymously via computers facilitates the expression of affective states such as emotions, opinions, doubts, risk, fears, etc. These affective states are generally repressed in more traditional communication contexts, such as face to face interviews or when people answer surveys. These resources are a gold mine for health professionals, giving them access to patient to patient exchanges, patient to health professional exchanges and even health professional to health professional exchanges.

\footnotetext{
${ }^{1}$ Funded by the MSH-M (Maison des Sciences de l'homme de Montpellier, trans. Humanities Home of Montpellier) and the inter-MSH network.

${ }^{2}$ http://www.healthforum.com/
} 
In literature, while many approaches have been proposed for text polarity analysis (positive, neutral and negative) [2], few ones focus on sentiment analysis (joy, anger, sadness, etc.) [3] or on the intensity of these two emotional states [4]. These approaches have been applied in various fields and can, for instance, be used to classify texts effectively. However, these methods rarely address the detection of targets or sources even if they provide relevant information. For example, lets consider the two following sentences: I'm afraid of my doctors reaction and My doctor is afraid of my reaction to the drug. Conventional methods detect that these two sentences are negative and that they contain the emotion fear. In the first case, they do not detect that this emotion involves the reaction of the doctor and that the fear is felt by the speaker. In the second case, they do not detect that the emotion deals with the patient's response to the drug and that the fear is felt by the doctor. In this paper, we focus on this level of precision in order to aggregate this type of information, in a second time, to multiple messages (e.g., find the number of messages in which patients express their fear about a drug). In literature, some methods analyze the sources of affective states (e.g., who feels the emotional state) [5], and others the targets (e.g., what is the object of the emotional state?) [6]. In this article, we focus on the target, in the specific context of the emotions expressed in health forums.

Our method aims to identify traces of emotions in texts associated with a target or a context, if possible medical ones, facilitating the interpretation of the emotion. This method can be generalized to other affective states and other fields of application. In this purpose, we suggest as $[7,8]$ do to incorporate the Shallow semantic parsing and use the FrameNet ${ }^{3}$ lexical resource. Based on the concept of semantic role defined by Baker and al. [9], this resource describes situations schematically. Annotation based on this resource allows us to identify in sentences expressions of emotions and to explain their components. We propose a typology of these annotations dedicated to our specific context. To our best knowledge, there is no method based on such type of annotations, customised thanks to a typology for health forum application field. We compare this approach to the more classic ones, which are based on a distance computation in the dependency tree of the sentences, to identify associations between emotions and predefined targets. Our method has been successfully evaluated on real posts.

The rest of this paper is organized as follows: in Section 2, we motivate our work in the framework of the semi-automatic analysis of health forums and we define the referred task of sentiment analysis. In Section 3, we show the latest methods dedicated to this task. In Section 4, we describe the conducted experiments, then the main results in Section 5. Finally, in Section 6, we conclude and give the main perspectives associated to this work.

\footnotetext{
$\overline{{ }^{3} \text { https://framenet.icsi.berkeley.edu/fndrupal/home }}$
} 


\section{Motivations and task definition}

As Siegrist pointed out [10], one of the greatest challenges for health professionals is to capture patients satisfaction. With this aim, he studied patients' feedback after their stay in the main American hospitals and turned them into raw data for decision making. Using the forums as an object of study, we are getting closer to the patients private sphere. Indeed, patients express things through posts they do not express in comments (even anonymous). However, precisely identifying the emotional state of patients through these messages is a difficult objective task and not always verifiable. However, we could consider using these large amounts of emotionally-charged texts to construct indicators that are relevant for health professionals. An example of such an application is We feel fine [11]. This tool scans the web with the aim of assessing users' moods. Every 10 minutes, the application considers sentences with emotional words and performs statistical calculations based on the type of feelings, age, gender, etc.

Sentiment analysis has been widely studied since the early 2000s. Many communities are interested in this area and their definitions and interpretations are highly varied (e.g., psychology, social sciences, computational linguistics, natural language processing, data mining, etc.). Sentiment analysis involves the extraction of emotional states expressed or implied in texts. There are several models of the opinion $[12,13]$, which vary according to the purpose of the study and the completed tasks. To generalize our work to any emotional state and any task, we consider the model described in Figure 1. Emotional state is experienced by a source (or experiencer). It refers to a polarity, that is to say, a judgment which can be positive if it is linked to a positive effect for the experiencer and in the opposite negative or in some cases neutral. The emotional state can also refer to an emotion such as anger, joy, sadness, etc. Generally, emotions are associated with a polarity. Joy is a positive example, anger is negative and surprise is neutral. Different levels of intensity can be associated to the emotional state (e.g., very successful, a little sad, etc.). Finally, the emotional state has a target which is the receptacle of the opinion or emotion.

In this work, we focus on target identification. They are generally present in texts as named-entities, events, abstract concepts, features associated with these abstract concepts or general contexts $[14,15]$. Lets consider the examples in Table 1. In S1, S2 and S3 sentences, the emotion fear is related to an entity represented respectively by the general concept drug, by the event beginning of the chemotherapy and by the named-entity IVEMED (which is a drug name). In $\mathrm{S} 4$, the target of the opinion involves one aspect, a characteristic, the tolerance rate to the drug. In S5, only the aspect is present. In S6, there is no explicit target and the target refers to the general context the sentence is formulated in. In S7, the target is detailed in the rest of the sentence and it is not limited to medical entity pain. Sometimes, it is difficult to distinguish the target from the circumstances which cause the emotional state. This is the case for example in S8. These examples illustrate the complexity of the task because targets can be expressed in very different ways. Unlike most of the approaches of the literature, 


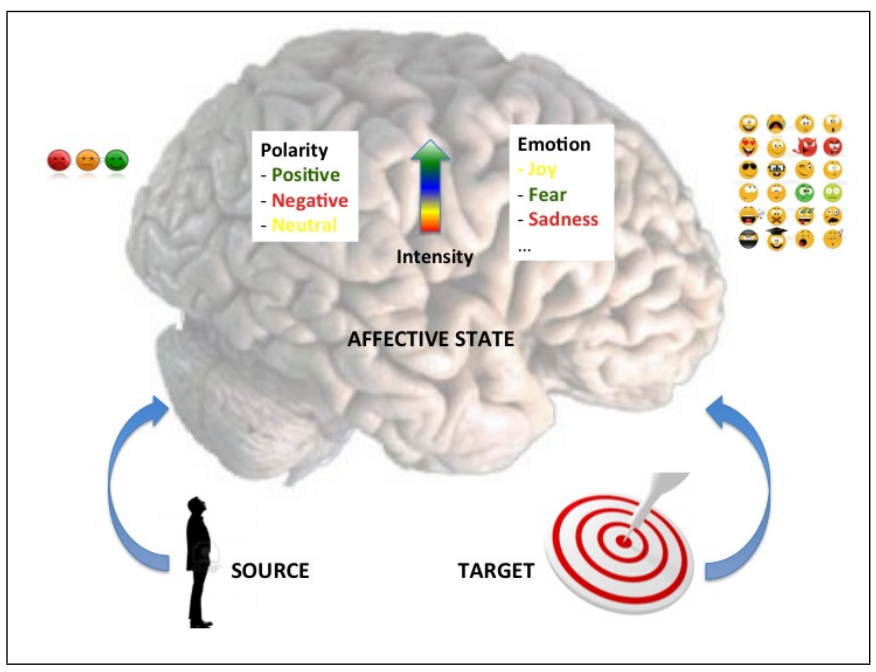

Fig. 1. Affective state model.

we take the position in this proposal not to limit the target to a few words, but rather to provide as much information as possible, like it is done in S7 and S8.

S1: I am afraid of this drug.

S2: I am afraid to begin the chemotherapy.

S3: I am afraid to take the IVEMEND.

S4: The rate of tolerance for this drug is excellent!

S5: The rate of tolerance is excellent.

S6: I am afraid.

S7: I'm afraid to live in pain for another ten years.

S8: I was given a 10 chance of living for 10 years, announced real prospects of recurrence and the fear stayed with me all this time.

Table 1. Examples of emotion expressions.

The (semi-)automatic analysis of forums is difficult from a technological standpoint. Most (semi)-automatic methods used in the health domain are applied to publications and hospital reports. Adapting these methods to messages from social media like forums is not at all simple. Such messages are written by patients in a quite loose style. They vary in size (between a hundred and a thousand characters). They contain non-standard grammatical structures, many misspellings, abbreviations, emotion-rich expressions as well as emotionrich words (I love, I hate), unconventional lay-out, e.g., repeated use of capital letters (TIRED), unconventional spelling (enoooooough), and punctuation repe- 
titions (!!!!!!!!), slang words that are (or not) specific to the forum or the topic (LOL vs. IVF) and emoticons (:-)). Message volumes are generally very high (in the French forum dedicated to breast cancer on Doctissimo website, there are more than 3, 300 threads, some of which containing more than 2, 000 replies). Finally, the processing of health forum data based on semi-automatic information extraction methods is a significant technological challenge. The method proposed in this paper is efficient without special pretraitement such as misspelling and grammar correction, which are difficult to implement generically in the case of health forum because of the non standardised vocabulary which varies a lot from a forum to another.

\section{State of the art}

Before associating an emotional state to a target, the first step consists in identifying in the texts some candidates for these two categories. To identify marks of emotions, many resources (list of words, phrases, idioms) are available. Most of these resources have been compiled for English texts and polarity analysis, e.g., General Inquirer [16], Linguistic Inquiry and Word Count [17], MicroWNOp [18]. More specific resources, such as the DAL dictionary [19] or the lexicon elaborated by Mohammad and Turney [20] were created for emotional words. There are also approaches for extending these vocabularies for specific application domains by building manual rules [21] or identifying co-occurring terms with words already identified as denoting emotions through large corpora or the web $[22$, 23]. There are also methods to identify emotions in text that are not limited to the use of lexicons such as $[24,25]$ which implement machine learning methods. To identify potential targets of opinions, approaches are generally specific to the application domain. Hu and Liu [26] used an association rules algorithm to identify common characteristics mentioned in products reviews. Zhuang and al. [27] used for movie reviews, annotated data and grammatical patterns. In the case of health forums, target identification is a priori more difficult because the authors discuss many entities which are hardly comparable and listable in advance as we have shown in the examples of Table 1.

Once candidates for opinions and candidates for targets are generated, two families of approaches can be used to connect them. The first one considers essentially linguistic aspects, represented as rules $[26,28]$ such as valence shifters (e.g., not, only, very, etc.) or conjunctions (e.g., the drug $\mathrm{x}$ is good but $\mathrm{Y}$ is better). These rules are very complex and some have been theorized in the context of the study of compositional semantics. Dowty and al. [29] consider that the meaning of an expression depends on the meaning of its components and composition rules. For example, my pain was reduced significantly is a positive expression composed of a negative term pain and a relationship significantly reduced. The effectiveness of this first family of methods is strongly related to language style that impacts on the linguistic rules to consider. In the context of health forums, they are difficult to implement because the styles vary from a forum to another. Although there are exceptions, in forums dealing with moth- 
erhood, addressing young women, the language is often familiar, close to SMS language while the language in forums dealing with back pain and addressing primarily elderly people is much more sustained. For our application, it is quite difficult to develop a generic method for any type of forums, relying solely on approaches based on lexicons and rules. The second family of methods is based on different distance computations between the emotional words and the potential targets. The most commonly used is the proximity: the chosen opinion candidate is the closest one to the target in number of words [26]. It is also possible to use the dependencies tree of a sentence. For example, Zhuang and al. [27] consider this tree as a graph and calculate the distance between a target candidate and an opinion candidate by a wide first search where the shortest path is calculated as the number of edges. Wu and al. [6] consider the distance in depth between the target candidate and the opinion candidate based on the lowest common parent in the dependencies tree. To improve the performance of these methods, some authors propose hybrid approaches and introduce linguistic rules for weighting the edges in the dependencies tree [30]. In our context, because of the langage pecularities (misspelling and gramatical errors...), we will show in the next section the importance of the robustness of the parser.

FEs Definition: The words in this frame describe the Experiencer's EMOTIONS with respect to some target. Although the target may refer to an actual, current state of affairs, quite often it refers to a general situation which causes the emotion. With certain verbs, the circumstances can also be expressed.

Annotations Examples:

My ENJOYMENT of the movie was considerably spoiled by the seven-foot-tall guy sitting in front of me.

Smithers takes great PLEASURE in collecting matchboxes.

I HATE it when you do that.

LUs Examples: abhor.v, abhorrence.n, abominate.v, adoration.n, adore.v, afraid.a, agape.a, antipathy.n, apprehensive.a, calm.a, comfort.n, compassion.n, , etc.

Table 2. Simplified example of the frame Experiencer Focus.

In this article, we propose to incorporate the Shallow semantic parsing not only to identify traces of emotions in health forums but also to improve targets detection. The principle is to associate semantic roles to different components of a statement such as Agent, Patient, Subject, etc. We are particularly interested in the resource developed in the framework of the FrameNet project [9]. These authors define Frames, corresponding to schematic representations of situations. Semantic roles, called Frame Elements (FE) are exclusively associated with frames. They are expressed by Lexical Units (LU). Table 2 shows a simplified example of the frame Experiencer Focus. This frame has two main FEs: the experiencer and the target. Another FE can also be used: the circumstances. As we can see in the annotations examples, the same FEs can be raised by 
constituents with different types of syntax and grammar. This theory has been successfully applied to automatic translation [31] and question/answer systems [32]. To our knowledge, only Kim and Hovy [8] have used the FrameNet resource for the identification of opinions. In this article, we suggest to handle specifically the case of emotions and we refine the notion of target used by these authors to distinguish different types of frames in order to better capture the expressed emotional states in relation with health issues.

\section{Initial evaluations}

Figure 2 describes the overall approach for identifying affective states and targets $^{4}$. It is divided into three steps:

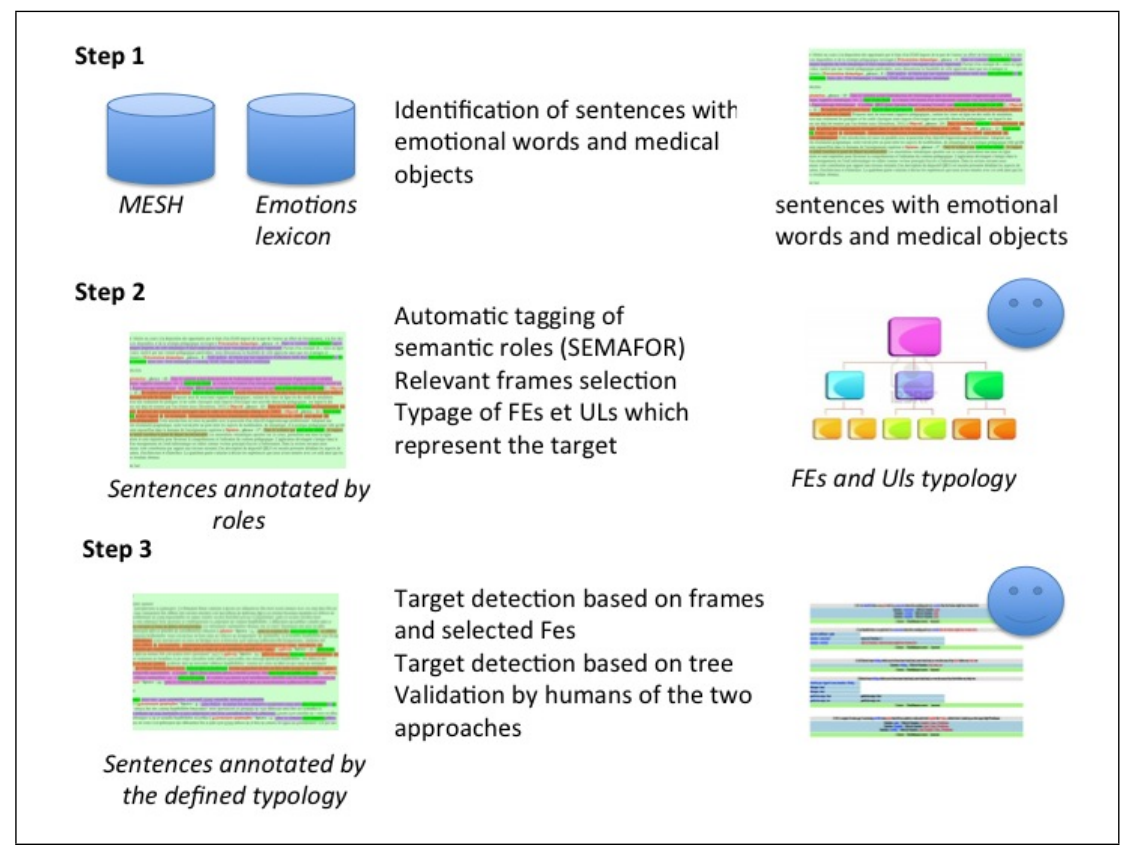

Fig. 2. Global approach.

Step 1: Corpus definition. We built a corpus from 17, 000 messages collected in the English-language Spine-health forum ${ }^{5}$. We automatically annotated the corpus with the vocabulary of emotions described in [20]. This lexicon consists in more than 14,000 entries characterized by their polarity and associated

\footnotetext{
${ }^{4}$ Data and annotated results are available at: http://tatoo.lirmm.fr/ pompidor/cgi/cicling2014.html

${ }^{5}$ http://www.spine-health.com/forum
} 
with 8 emotions. In this work, we consider only 6 emotions [33]: anger, disgust, fear, joy, sadness and surprise. This automatic annotation enabled us to filter $22 \%$ of the messages (not containing emotional words). In order to focus only on emotions associated with medical items, we used the classical medical thesaurus $\mathrm{MeSH}^{6}$ to identify medical units, which allowed us to filter messages without any medical references ( $6 \%$ of messages). In a message, more than 6 emotions were usually expressed because the messages were relatively long. We therefore chose to segment the messages in sentences. We finally kept 1,000 sentences.

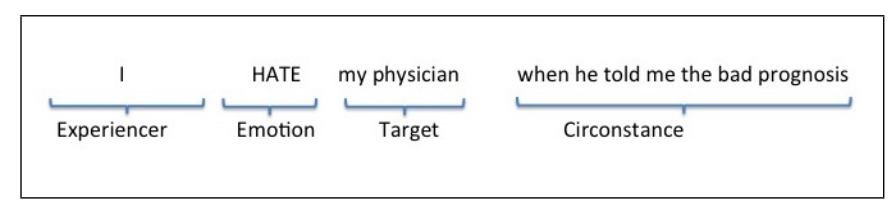

Fig. 3. Expression of emotions.

Step 2: Identification of relevant frames for the emotions target detection. We have used the SEMAFOR tool [34] to annotate texts according to the elements provided by the FrameNet ressources. Figure 3 shows an example of the result. We studied the frames identified by this tool and chose manually those that are relevant to the target identification in the medical context. Among the existing 1164 frames, we selected 16 ones relative to the expression of emotional states, 7 explaining generally the expression of these emotional states and 5 which are specific to medical objects. For each frame, we chose the FEs that play the role of emotion or target. For example, for the frame FEAR, we used the FE expressor as emotion and topic as target. Table 3 lists these frames.

Step 3: Evaluation. In the initial corpus, we kept only the sentences corresponding to the frames selected at the second step (i.e. 345 sentences). We transformed the output of the SEMAFOR tool according to the typology described in Table 3. We used one color to reveal emotions and another one for the context and any element that can help for interpreting the emotion. Finally, the output presented to the users corresponds to a list of typed information. We call this approach the Roles approach. We also suggested for each sentence a relationship between emotions and medical objects based on the distance computation in the dependencies tree. To do this, we used the morphosyntactic tagger developed at Sandford ${ }^{7}$. To determine the distance between targets and emotion candidates, we chose the computation based on the shortest path in number of edges that had be proven to be the most effective (see Section 3). The output presented to the user corresponds to couples Emotion - Medical Unit. We call this approach the Path approach. The results of these two approaches were validated via a web interface (see Figure 4) used by 10 experts to verify if the information was:

\footnotetext{
${ }^{6}$ http://www.ncbi.nlm.nih.gov/mesh

${ }^{7}$ http://nlp.stanford.edu/software/lex-parser.shtml
} 


\begin{tabular}{|l|l|}
\hline Emotions & Targets explaining emotions \\
\hline Experiencer focus & General context \\
Experiencer obj & Mental stimulus exp focus \\
Emotions & Partiality \\
Emotion active & Activity start \\
Emotion directed & Causation \\
Emotion heat & Awareness \\
Emotions by stimulus & \\
Emotions success or failure & Medical context \\
Complaining & Medical conditions \\
Contrition & Observable body parts \\
Desirability & Perception body \\
Desiring : Event & Cause harm \\
Fear & Intoxicants \\
Feeling & Cure \\
Sensation & Experience bodily harm \\
Tolerating & \\
\hline
\end{tabular}

Table 3. Typology of frames of interest manually identified.

correct, partially correct (if a part of the target had been forgotten or added) or incorrect. At least three experts answered this question for each sentence.

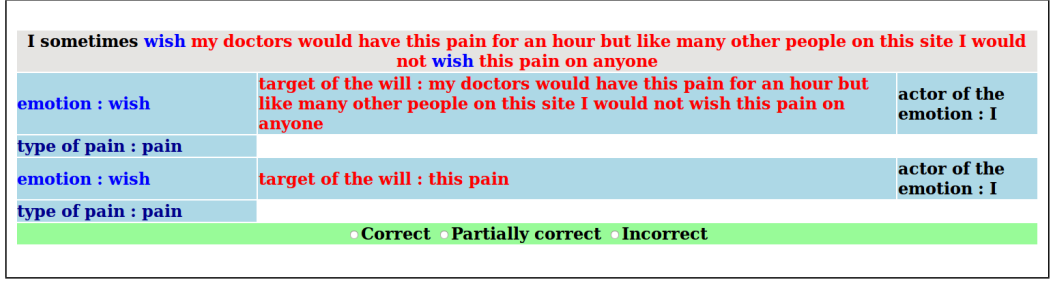

Fig. 4. Validation interface.

\section{$5 \quad$ Results}

Table 4 presents the results obtained from the methodology described in Section 4. We studied the agreement between annotators using the Fleiss Kappa measure $^{8}$ and a measure based on Regular Equivalence Classes (REC) and obtained in both cases a moderate agreement.

As might be expected, the Path Approach is the least effective approach. The main reason is due to the fact that in our case study and contrary to other

\footnotetext{
${ }^{8}$ http://en.wikipedia.org/wiki/Fleisskappa
} 
application domains, it is not possible to define in advance a comprehensive list of targets. For example, when studying opinions about products, targets are related to the products themselves or to their characteristics that can be easily identified because they appear frequently in the comments. In the forums, the targets are really diversified and they are not limited to medical objects identified using the MeSH. For example, in the sentence I fear the long term tendency, the Path Approach identifies fear as the emotion but it will not be able to associate it with tendency which is not an entry in the MeSH. A second limitation is related to the parser performances used to extract the dependency tree on the forums sentences because they are often poorly constructed (punctuation, spelling, etc.). The SEMAFOR parser used in the Role approach is more robust for langage pecularities. Finally, in step 2, the Role Approach efficiently excludes sentences (not excluded if we use only the Path approach) that do not contain emotion expressions in the context of health forums. They have been selected in the initial corpus because of the presence of a word of emotion issued from a general resource, which is not always representative in the specific medical context. For example, the sentence If you want your curve progression halted, it can only be done by surgery was chosen because of the presence of the word progression in the general emotions resource, which is not relevant in our context.

Roles Approach is the most effective one, especially when emotion is carried by a verb (e.g., I fear the surgeon will be reluctant to continue helping control my pain). However, it shows some limitations. The study of sentences for which human annotations were different, shows that the targets identified as relating to health are easier to interpret than the general targets. For example, I hope that your injection starts to bring you raised soon is simple to analyze. Moreover, the FrameNet resource does not fully integrate all emotional states we aim to identify in texts. For example, only two frames identify the uncertainty (Certainty, Degree) which are particularly interesting to consider in health forums. Finally, the generalization of this method to other languages is difficult. There is no resource, for French, as complete as FrameNet.

\begin{tabular}{|c|c|c|c|c|c|}
\hline & \multicolumn{5}{|c|}{ Correct Partially correct Incorrect Fleiss Kappa measure REC measure } \\
\hline Role A & $8 \%$ & $43 \%$ & $49 \%$ & 0.48 (Moderate) & 0.52 \\
\hline Path Approch & $53 \%$ & $34 \%$ & $13 \%$ & 0.58 (Moderate) & 0.63 \\
\hline
\end{tabular}

Table 4. Results

\section{Conclusions and perspectives}

Forums represent a large and diverse base of knowledge about patients' perceptions of their illness and the care that are eventually provided to them. In this article, we described an approach to help a reader to identify traces of emotions 
in the health forum and to explain their components. We have shown that the use of a semantic roles tagger is quite effective to interpret these targets without any pretraitement of the messages.

Prospects associated with this work are numerous. First, in this work we focus only on the targets expressed in sentences and we now have to focus on inter-sentence relationships at paragraph or message level. In addition, identifying the opinion holder could represent some additional information that would be relevant for the forums analysis. It would be quite simple to capture this information as it is already present in the elements returned by the semantic roles tagger. Contrary to the analysis of products reviews that usually contain only the feelings of the commentators, patients in the forums relate emotions that are not their own (e.g., my doctor is concerned that my blood glycerol increases). Moreover, once the relationship between emotion and target is identified, it can be generalized to a set of messages in order to summarize the emotional states of different patients about a specific target. For example, in the case of the analysis is associated with a particular emotions treatment, characteristics associated with this medical purpose are well known (price, tolerance, side effects, etc.). It is then possible to make associations between target and opinion as it is done in the case of movies or products reviews [26, 27].

Finally, the main limitation of this contribution is to restrict emotion identification only to the case in which an emotional word is present. As pointed out by [35], in most cases people express emotions implicitly without using these emotional words. An emotion can be limited to something a person feels about a fact and not the sentiment that a person expresses about this fact. Thus, it could be common to explicitly express sentiments about things, but it is more common to feel emotions without expressing them explicitly. In future work, we should take into account this fact and try to identify emotions beyond the explicit cases, because it is possible that in such implicit cases the identification of targets is different.

\section{References}

1. Hancock, J.T., Toma, C.L., Ellison, N.B.: The truth about lying in online dating profiles. In Rosson, M.B., Gilmore, D.J., eds.: CHI, ACM (2007) 449-452

2. Pang, B., Lee, L., Vaithyanathan, S.: Thumbs up?: Sentiment classification using machine learning techniques. In: Proceedings of the ACL-02 Conference on Empirical Methods in Natural Language Processing - Volume 10. EMNLP '02, Stroudsburg, PA, USA, Association for Computational Linguistics (2002) 79-86

3. Strapparava, C., Mihalcea, R.: Learning to identify emotions in text. In: Symposium on Applied Computing, New York, NY, USA, ACM (2008) 1556-1560

4. Wiebe, J.: Learning subjective adjectives from corpora. In: Proceedings of the Seventeenth National Conference on Artificial Intelligence and Twelfth Conference on Innovative Applications of Artificial Intelligence, AAAI Press (2000) 735-740

5. Choi, Y., Cardie, C., Riloff, E., Patwardhan, S.: Identifying sources of opinions with conditional random fields and extraction patterns. In: Proceedings of the Human Language Technology Conference and the Conference on Empirical Methods in Natural Language Processing (HLT/EMNLP). (2005) 
6. Wu, Y., Zhang, Q., Huang, X., Wu, L.: Phrase dependency parsing for opinion mining. In: Proceedings of the 2009 Conference on Empirical Methods in Natural Language Processing: Volume 3 - Volume 3. EMNLP '09, Stroudsburg, PA, USA, Association for Computational Linguistics (2009) 1533-1541

7. Ruppenhofer, J., Somasundaran, S., Wiebe, J.: Finding the sources and targets of subjective expressions. In Calzolari, N., Choukri, K., Maegaard, B., Mariani, J., Odjik, J., Piperidis, S., Tapias, D., eds.: Proceedings of the Sixth International Language Resources and Evaluation (LREC'08), Marrakech, Morocco, European Language Resources Association (ELRA) (May 2008) http://www.lrecconf.org/proceedings/lrec2008/.

8. Kim, S.M., Hovy, E.: Extracting opinions, opinion holders, and topics expressed in online news media text. In: Proceedings of the Workshop on Sentiment and Subjectivity in Text. SST '06, Stroudsburg, PA, USA, Association for Computational Linguistics (2006) 1-8

9. Baker, C.F., Fillmore, C.J., Lowe, J.B.: The Berkeley FrameNet project. In: Proceedings of COLING/ACL. (1998) 86-90

10. Siegrist, J.: Emotions and Health in Occupational Life: New Scientific Findings and Policy Implications : Inauguration Speech Belle Van Zuylen Professorship. Universiteit Utrecht (1994)

11. Kamvar, S.D., Harris, J.: We feel fine and searching the emotional web. In: ACM International conference on Web search and data mining, New York, NY, USA, ACM (2011) 117-126

12. Kim, S.M., Hovy, E.: Determining the sentiment of opinions. In: Proceedings of the International Conference on Computational Linguistics (COLING). (2004) $1367-1373$

13. Kobayashi, N., Inui, K., Matsumoto, Y.: Extracting aspect-evaluation and aspectof relations in opinion mining. In: Proceedings of the 2007 Joint Conference on Empirical Methods in Natural Language Processing and Computational Natural Language Learning (EMNLP-CoNLL. (2007) 1065-1074

14. Popescu, A.M., Etzioni, O.: Extracting product features and opinions from reviews. In: Proceedings of the Conference on Human Language Technology and Empirical Methods in Natural Language Processing. HLT '05, Stroudsburg, PA, USA, Association for Computational Linguistics (2005) 339-346

15. Wilson, T., Wiebe, J., Hoffmann, P.: Recognizing contextual polarity in phraselevel sentiment analysis. In: Proceedings of the Human Language Technology Conference and the Conference on Empirical Methods in Natural Language Processing (HLT/EMNLP). (2005) 347-354

16. Stone, P.J., Hunt, E.B.: A Computer Approach to Content Analysis: Studies Using the General Inquirer System. AFIPS '63 (Spring). ACM, New York, NY, USA (1963)

17. Tausczik, Y.R., Pennebaker, J.W.: The psychological meaning of words: Liwc and computerized text analysis methods. Volume 29. (2010) 24-54

18. Cerini, S., Compagnoni, V., Demontis, A., Formentelli, M., Gandini, G. In: Language resources and linguistic theory: Typology, second language acquisition, English linguistics., Milano, IT, Franco Angeli Editore (2007)

19. Whissell, C.: The dictionary of affect in language. Academic Press (1989)

20. Mohammad, S.M., Turney, P.D.: Emotions Evoked by Common Words and Phrases : Using Mechanical Turk to Create an Emotion Lexicon. In: Workshop on Computational Approaches to Analysis and Generation of Emotion in Text, Stroudsburg, PA, USA, ACL (2010) 26-34 
21. Neviarouskaya, A., Prendinger, H., Ishizuka, M.: Affect analysis model: Novel rule-based approach to affect sensing from text. Volume 17., New York, NY, USA, Cambridge University Press (January 2011) 95-135

22. Harb, A., Plantié, M., Dray, G., Roche, M., Trousset, F., Poncelet, P.: Web Opinion Mining : How to extract opinions from blogs ? Categories and Subject Descriptors. In: International conference on Soft Computing as Transdisciplinary Science and Technology. (2008) 211-217

23. Kozareva, Z., Navarro, B., Vazquez, S., Montoyo, A.: UA-ZBSA: a headline emotion classification through web information. In: 4th International Workshop on Semantic Evaluations, Stroudsburg, PA, USA, ACL (2007) 334-337

24. Strapparava, C., Mihalcea, R.: Learning to identify emotions in text. In: Proceedings of the 2008 ACM Symposium on Applied Computing. SAC '08, New York, NY, USA, ACM (2008) 1556-1560

25. Pardo, F.M.R., Rosso, P.: On the identification of emotions and authors' gender in facebook comments on the basis of their writing style. In: Proceedings of the First International Workshop on Emotion and Sentiment in Social and Expressive Media: approaches and perspectives from AI (ESSEM 2013) A workshop of the XIII International Conference of the Italian Association for Artificial Intelligence (AI*IA 2013), Turin, Italy, December 3, 2013. (2013) 34-46

26. Hu, M., Liu, B.: Mining opinion features in customer reviews. In: Proceedings of the 19th National Conference on Artifical Intelligence. AAAI'04, AAAI Press (2004) $755-760$

27. Zhuang, L., Jing, F., Zhu, X.Y.: Movie review mining and summarization. In: Proceedings of the 15th ACM International Conference on Information and Knowledge Management. CIKM '06, New York, NY, USA, ACM (2006) 43-50

28. Mudinas, A., Zhang, D., Levene, M.: Combining lexicon and learning based approaches for concept-level sentiment analysis. In: Proceedings of the First International Workshop on Issues of Sentiment Discovery and Opinion Mining. WISDOM '12, New York, NY, USA, ACM (2012) 5:1-5:8

29. Dowty, D.R., Wall, R.E., Peters, S.: Introduction to Montague Semantics. Volume 11. D. Reidel, Dordrecht (1989)

30. Ding, X., Liu, B.: The utility of linguistic rules in opinion mining. In: Proceedings of the 30th Annual International ACM SIGIR Conference on Research and Development in Information Retrieval. SIGIR '07, New York, NY, USA, ACM (2007) $811-812$

31. Boas, H.C.: Bilingual FrameNet dictionaries for machine translation. In Rodríguez, M.G., Araujo, C.P.S., eds.: Proceedings of the Third International Conference on Language Resources and Evaluation. Volume IV., Las Palmas (2002) 1364-1371

32. Narayanan, S., Harabagiu, S.: Question answering based on semantic structures. In: Proceedings of the 20th International Conference on Computational Linguistics. COLING '04, Stroudsburg, PA, USA, Association for Computational Linguistics (2004)

33. Ekman, P.: An argument for basic emotions. Volume 6. (1992) 169-200

34. Das, D., Schneider, N., Chen, D., Smith, N.A.: Probabilistic frame-semantic parsing. In: Human Language Technologies: The 2010 Annual Conference of the North American Chapter of the Association for Computational Linguistics. HLT '10, Stroudsburg, PA, USA, Association for Computational Linguistics (2010) 948-956

35. Osherenko, A., André, E.: Lexical affect sensing: Are affect dictionaries necessary to analyze affect? In: Proceedings of the $2 \mathrm{Nd}$ International Conference on Affective Computing and Intelligent Interaction. ACII '07, Berlin, Heidelberg, SpringerVerlag (2007) 230-241 